\title{
Coenzyme A Sequestration in Rat Hearts Oxidizing Ketone Bodies
}

\author{
Raymond R. Russell III and Heinrich Taegtmeyer \\ Department of Medicine, Division of Cardiology, University of Texas Medical School at Houston, Houston, Texas 77030
}

\begin{abstract}
Previous studies have indicated that ketone body-mediated contractile failure in rat hearts is due to inhibition of 2-oxoglutarate dehydrogenase, and it has been speculated that this inhibition is due to the sequestration of intramitochondrial $\mathrm{CoA}$ as acetoacetyl-CoA and acetyl-CoA. These studies were performed to determine whether oxidation of acetoacetate by isolated rat heart mitochondria results in a fall in intramitochondrial nonesterified CoA [CoASH] and whether increasing the available CoA improves contractile performance in hearts oxidizing acetoacetate. The oxidation of acetoacetate by isolated rat heart mitochondria resulted in depressed state 3 respiration as well as in a decrease in [CoASH]. Increasing the tissue content of CoASH in perfused hearts by providing the precursors for $\mathrm{CoA}$ relieved inhibition of 2-oxoglutarate dehydrogenase and improved the contractile performance of isolated working hearts. In contrast, the addition of carnitine increased the tissue content of CoASH but did not improve function. These findings suggest the presence of two different pools of CoASH. We conclude that ketone body-mediated inhibition of 2-oxoglutarate dehydrogenase is due to decreased intramitochondrial CoASH and that this inhibition of the citric acid cycle is a plausible mechanism for concomitant contractile failure. ( $J$. Clin. Invest. 1992. 89:968-973.) Key words: acetoacetate • 2oxoglutarate dehydrogenase $\bullet$ citric acid cycle $\bullet$ rat heart
\end{abstract}

\section{Introduction}

Oxidation of ketone bodies as a sole substrate by the isolated working rat heart results in reversible contractile failure $(1,2)$, despite the ability of mammalian hearts to utilize ketone bodies as fuel for respiration (3). We (1) and others $(4,5)$ have previously suggested that the failure results from inhibition of the citric acid cycle at the level of 2-oxoglutarate dehydrogenase through the sequestration of the required cofactor nonesteri-. fied $\mathrm{CoA}(\mathrm{CoASH})^{1}$ as the thioesters acetoacetyl-CoA and acetyl-CoA. Measurements of the whole tissue content of CoASH and acetyl-CoA from hearts oxidizing ketone bodies are consistent with this mechanism $(1,2)$, although the intramitochondrial [CoASH] has not been determined in hearts oxidizing ketone bodies.

A portion of the data in this article was presented in abstract form at the 64th Scientific Sessions of the American Heart Association, 11-14 November 1991.

Address correspondence to Dr. Heinrich Taegtmeyer, Division of Cardiology, University of Texas Medical School at Houston, 6431 Fannin, Houston, TX 77030. 1991.

Received for publication 25 June 1991 and in revised form 4 October

1. Abbreviation used in this paper: CoASH, nonesterified CoA.

J. Clin. Invest.

(c) The American Society for Clinical Investigation, Inc.

0021-9738/92/03/0968/06 \$2.00

Volume 89, March 1992, 968-973
Because the above findings suggest that free CoASH may be rate limiting for the 2-oxoglutarate dehydrogenase reaction, it seemed logical to assume that increasing the supply of CoASH may improve contractile function in hearts oxidizing acetoacetate. The tissue content of CoASH can be increased in one of two ways. First, the relative distribution of $\mathrm{CoA}$ between nonesterified CoASH and esterified CoA (e.g., acetyl-CoA, fatty acyl-CoA) can be altered by the provision of $L$-carnitine, which has been shown to result in the transfer of acetyl units from acetyl-CoA and acetoacetyl-CoA to carnitine via the action of the enzyme carnitine acetyltransferase $(4,6)$, thereby forming acetylcarnitine and acetoacetylcarnitine while increasing the tissue content of CoASH. This is supported by the observations that the addition of carnitine to isolated rat heart mitochondria oxidizing acetoacetate resulted in an increase in state 3 respiration $(4,5)$ and that the incubation of isolated mitochondria with carnitine increases levels of intramitochondrial CoASH and decreases levels of acetyl-CoA (6). Because carnitine is readily transported across the sarcolemma (7), it may be possible to increase intracellular levels of carnitine in the intact heart in an attempt to "buffer" acetyl-CoA and acetoacetyl-CoA as the carnitine esters acetylcarnitine and acetoacetylcarnitine.

The second manner in which the tissue content of CoASH can be elevated relies on the increased synthesis of CoA by providing exogenous sources of the precursors of CoA, pantothenic acid and cysteine (8). The provision of these precursors without any exogenous energy-providing substrate has been shown to result in a 50\% increase in the tissue content of total CoA (9). It has previously been demonstrated that the enrichment of the CoA pool decreases the oxidation of palmitate and increases the incorporation of palmitate into triacylglycerols (9), but the effect of elevated levels of CoA on ketone body metabolism is not known.

It was, therefore, the goal of this study to determine, first, whether the metabolism of ketone bodies results in a fall in intramitochondrial concentrations of $\mathrm{CoASH}$ and, second, whether measures aimed at increasing the myocardial content of CoASH are effective in improving flux through 2-oxoglutarate dehydrogenase and thereby improving contractile function in hearts oxidizing ketone bodies.

\section{Methods}

Heart perfusions. Rat hearts were perfused in the working rat heart perfusion apparatus previously described $(10)$ with oxygenated $(95 \%$ $\mathrm{O}_{2} / 5 \% \mathrm{CO}_{2}$ ) Krebs-Henseleit bicarbonate buffer at $37^{\circ} \mathrm{C}$ for the times specified in the figures and tables. During the perfusion period, aortic systolic and diastolic pressures and cardiac output were monitored and used to calculate the pressure-volume work performed by the heart (10). After perfusion, hearts were freeze clamped while still beating on the cannula and stored in liquid nitrogen until extracted for assay (1).

To increase the tissue content of CoASH and total CoA, a group of hearts was preperfused in a retrograde fashion (11) for $45 \mathrm{~min}$ at a perfusion pressure of $60 \mathrm{mmHg}$ with pantothenic acid $(15 \mu \mathrm{M})$, cysteine $(0.1 \mathrm{mM})$, and DTT $(0.2 \mathrm{mM})(8)$. DTT was added to prevent the 
oxidation of cysteine to cystine and thereby to maximize the synthesis of $\mathrm{CoA}(12)$.

Determination of intramitochondrial $\mathrm{CoASH}, \mathrm{NAD}^{+}$, and 2-oxoglutarate. Mitochondria were isolated from the hearts of adult, male Sprague-Dawley rats using the Nagarse method of Idell-Wenger et al. (13). Mitochondria ( $8 \mathrm{mg}$ mitochondrial protein) were incubated at $25^{\circ} \mathrm{C}$ in sucrose $(200 \mathrm{mM}), 3-(N$-morpholino)propanesulfonic acid (MOPS; $10 \mathrm{mM})$, and potassium phosphate $(10 \mathrm{mM}, \mathrm{pH} 7.4)$ containing ADP (320 nM), glucose ( $50 \mathrm{mM})$, hexokinase (40 U), dextran (60 $\mathrm{mg} / \mathrm{ml})$, and malate $(5 \mathrm{mM})$. In addition to the above, either acetoacetate $(5 \mathrm{mM})$ or pyruvate $(5 \mathrm{mM})$ was added as a substrate. Glucose and hexokinase were added as a regenerating system for extramitochondrial ADP, and dextran was added to enhance sedimentation of mitochondria during centrifugation (14). To determine extramitochondrial sucrose space and intramitochondrial volume, respectively, $\left[\mathrm{U}-{ }^{14} \mathrm{C}\right]-$ sucrose and ${ }^{3} \mathrm{H}_{2} \mathrm{O}$ were added to the incubation media.

The mitochondrial suspensions were incubated for $5 \mathrm{~min}$ in a microcentrifuge tube (Eppendorf Inc., Fremont, CA) containing a layer of silicone oil overlying $500 \mu \mathrm{l}$ of perchloric acid $(16 \% \mathrm{wt} / \mathrm{vol})$. After the 5 -min incubation period, the tubes were immediately centrifuged for $30 \mathrm{~s}$ in a benchtop microcentrifuge, sedimenting the mitochondria through the silicone oil and into the perchloric acid. The incubation medium was then removed and acidified.

Both the acidified mitochondrial extracts and the incubation media were neutralized and assayed fluorimetrically for $\mathrm{CoASH}, \mathrm{NAD}^{+}$, and 2-oxoglutarate (15). The intramitochondrial content of each of the metabolites was determined by correcting the metabolite content of the mitochondrial extracts for the metabolite content of incubation media trapped in the extramitochondrial space (16). Oxygen consumption was determined for mitochondria oxidizing either pyruvate $(5 \mathrm{mM})$ or acetoacetate $(5 \mathrm{mM})$ in media containing sucrose $(250 \mathrm{mM}), 3-(N$ morpholino)propanesulfonic acid (MOPS; $10 \mathrm{mM}$ ), potassium phosphate $(10 \mathrm{mM})$, malate $(5 \mathrm{mM})$, and ADP $(320 \mathrm{nM})$ by the method of Chance and Williams (17). Mitochondrial protein content was determined by the Biuret reaction (18) after mitochondrial samples were solubilized by the addition of deoxycholate to a final concentration of $1 \%$.

Tissue metabolite analysis. Fluorimetric determinations of $\mathrm{CoASH}$, acetyl-CoA, 3-ketoacyl-CoA derivatives (which includes acetoacetyl-CoA), acetylcarnitine, 3-ketoacylcarnitine, 2-oxoglutarate, glutamate, and succinyl-CoA were performed on perchloric acid extracts of frozen tissue samples (15). Because the enzyme used in the determination of acetoacetyl-CoA, $\beta$-hydroxyacyl-CoA dehydrogenase, has very little chain length specificity, the results of this assay represent the total acid-soluble 3-ketoacyl-CoA pool. Total CoA was determined fluorimetrically on extracts from tissue samples hydrolyzed with ethanolic $\mathrm{KOH}(15)$. The values for all tissue metabolites are expressed as micromoles per gram dry weight. All values are reported as the mean \pm SEM.

\section{Results}

Table I lists the intramitochondrial concentrations of CoASH, 2-oxoglutarate, and $\mathrm{NAD}^{+}$for mitochondria oxidizing either pyruvate or acetoacetate in the presence of malate. The state 3 rate of respiration for mitochondria using acetoacetate was approximately half that for mitochondria oxidizing pyruvate, which is consistent with inhibition of 2-oxoglutarate dehydrogenase (19). After $5 \mathrm{~min}$ of incubation with acetoacetate and malate, the intramitochondrial [CoASH] was $43 \%$ of that for mitochondria oxidizing pyruvate and in the same order of magnitude as the value for the $K_{\mathrm{mCoA}}$ reported by Read et al. (20) for rat heart 2-oxoglutarate dehydrogenase. Changes in the intramitochondrial 2-oxoglutarate concentration mirrored the changes seen in isolated working rat hearts oxidizing acetoacetate $(1,2)$ and were consistent with the accumulation of 2-oxoglutarate. In addition, the intramitochondrial concentration of $\mathrm{NAD}^{+}$was increased in mitochondria oxidizing acetoacetate compared with mitochondria oxidizing pyruvate. Taken together, these findings are consistent with decreased state 3 respiration, and therefore decreased energy production, that results from inhibition of 2-oxoglutarate dehydrogenase. The fall in the intramitochondrial [CoASH] with the oxidation of acetoacetate would indicate that this inhibition is associated with the sequestration of $\mathrm{CoASH}$.

Because inhibition of 2-oxoglutarate dehydrogenase and ultimately depressed contractile performance are both associated with the fall in both the whole tissue content of CoASH $(1,2)$ and the intramitochondrial [CoASH] (Table I), the provision of greater amounts of CoASH should improve performance in hearts oxidizing ketone bodies. Fig. 1 represents the cardiac output and pressure-volume work after $60 \mathrm{~min}$ of perfusion for hearts perfused with either acetoacetate or acetoacetate plus carnitine (preload: $15 \mathrm{~cm} \mathrm{H}_{2} \mathrm{O}$, afterload: $140 \mathrm{~cm} \mathrm{H}_{2} \mathrm{O}$ ). As expected, there was a dramatic and rapid fall in both cardiac output and pressure-volume work for hearts using acetoacetate as the sole substrate. Although hearts oxidizing acetoacetate in the presence of carnitine had a greater initial cardiac output and pressure-volume work, the decline in both cardiac output and pressure-volume work was similar to that for hearts oxidizing acetoacetate alone. In addition, increasing the concentration of carnitine in the perfusate (from 0.5 to $5 \mathrm{mM}$ ) did not improve function (data not presented).

Because the addition of carnitine to hearts oxidizing acetoacetate failed to improve contractile function, as would be predicted by the mitochondrial studies cited earlier $(4,5)$, it was important to determine whether carnitine altered the distribution of $\mathrm{C} 2$ and $\mathrm{C} 4$ units between their $\mathrm{CoA}$ and carnitine derivatives. Despite the decline in contractile function for hearts perfused with carnitine, there was a $24 \%$ increase in the tissue level of acetylcarnitine and an eightfold increase in the level of 3-ketoacylcarnitine (including acetoacetylcarnitine) in hearts perfused with acetoacetate plus carnitine compared with hearts perfused with acetoacetate alone (Table II). In addition, there

Table I. Intramitochondrial Concentrations of CoASH, 2-Oxoglutarate, and $\mathrm{NAD}^{+}$for Mitochondria Oxidizing Either Pyruvate or Acetoacetate

\begin{tabular}{lcccc}
\hline \multicolumn{1}{c}{ Substrate } & State 3 respiration & CoASH & 2-Oxoglutarate & NAD \\
\hline & natoms $O_{2} /$ min per $m g$ & & $m M$ \\
Pyruvate $(5 \mathrm{mM})+$ malate $(5 \mathrm{mM})$ & $292.0 \pm 17.8$ & $0.108 \pm 0.022$ & $0.128 \pm 0.012$ & $0.187 \pm 0.011$ \\
Acetoacetate $(5 \mathrm{mM})+$ malate $(5 \mathrm{mM})$ & $151.7 \pm 25.3^{*}$ & $0.046 \pm 0.011^{*}$ & $0.205 \pm 0.017^{*}$, & $0.409 \pm 0.095^{*}$ \\
\hline
\end{tabular}

Values are means \pm SEM for four hearts. ${ }^{*} P<0.001$. See text for further details. 

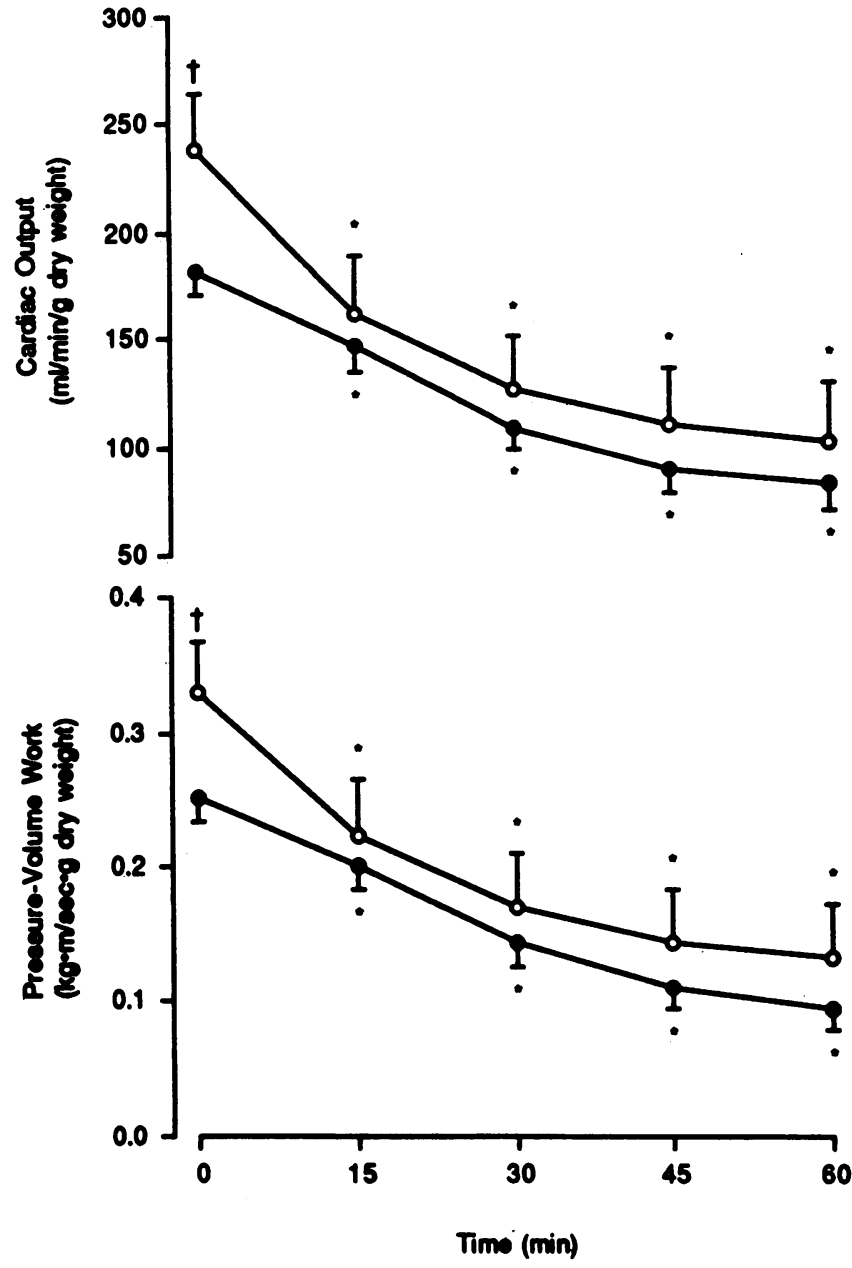

Figure 1. Cardiac output and pressure-volume work for working rat hearts $(n=10)$ oxidizing acetoacetate $(5 \mathrm{mM})$ either alone $(0)$ or with L-carnitine ( $5 \mathrm{mM} ; \bullet)$. ${ }^{*} P<0.05$ compared with time zero for the respective group; ${ }^{\dagger} P<0.05$ compared with time zero for hearts oxidizing acetoacetate alone.

was a 70\% decrease in acetyl-CoA in hearts using acetoacetate in the presence of carnitine compared with hearts oxidizing acetoacetate alone. Unexpectedly, there was a small increase in the amount of 3-ketoacyl-CoA in hearts that were perfused with carnitine, although the ratio of 3-ketoacyl-CoA/3-ketoacylcarnitine fell ( 2.20 vs. 0.38 for hearts perfused with acetoacetate alone or acetoacetate plus carnitine, respectively). The increase in 3-ketoacyl-CoA may represent an increase in longer chain fatty acid entry into the mitochondria stimulated by cyto- solic carnitine. Along with the changes in the levels of the acyl derivatives of $\mathrm{CoA}$, there was a $57 \%$ increase in the amount of free CoASH in hearts perfused with acetoacetate and carnitine compared with hearts using acetoacetate alone. These changes are consistent with an increase in the myocardial content of available carnitine and the transfer of $\mathrm{C} 2$ and $\mathrm{C} 4$ units from CoA to carnitine.

While the addition of carnitine did not improve function for hearts oxidizing acetoacetate, it has been suggested by others that the exchange between carnitine and acetylcarnitine is metabolically "sluggish," with a low flux of acyl groups (21), and that elevated levels of extramitochondrial carnitine do not affect intramitochondrial levels of acetyl-CoA (22). It was therefore necessary to attempt to increase the intramitochondrial [CoASH] through other means. It has previously been demonstrated that perfusing rat hearts initially at low workload with the precursors of $\mathrm{CoA}$ increases the tissue content of total CoA (8). In these studies, retrograde preperfusion of hearts for 45 min with pantothenic acid $(15 \mu \mathrm{M})$, cysteine $(0.1 \mathrm{mM})$, and DTT $(0.2 \mathrm{mM})$ resulted in a significant increase in the tissue content of both total CoA and CoASH immediately after the Langendorff perfusion period compared with hearts retrogradely perfused with no CoA precursors (Table III). This preperfusion technique was, therefore, used to increase the tissue content of available CoASH in an attempt to provide the intramitochondrial CoASH necessary to support continued flux through 2-oxoglutarate dehydrogenase, thereby improving contractile function.

After the retrograde preperfusion period, hearts were immediately switched over to the working (anterograde) heart circuit and perfused for $30 \mathrm{~min}$ with acetoacetate $(5 \mathrm{mM})$. There was no change in either the coronary flow or heart rate for either group during the preperfusion period (data not presented). Cardiac output and pressure-volume work were similar for hearts preperfused with CoA precursors and hearts preperfused with no exogenous precursors during the first $10 \mathrm{~min}$ of perfusion (Fig. 2). However, by $20 \mathrm{~min}$ of perfusion with acetoacetate, cardiac output had fallen to $62 \%$ of the initial value for hearts preperfused with no CoA precursors, whereas hearts preperfused with CoA precursors had a cardiac output that was $92 \%$ of the initial value. Similarly, the pressure-volume work after 20 minutes of perfusion with acetoacetate was 64 and 86\%, respectively, for hearts preperfused without any substrate and hearts preperfused with CoA precursors. After 30 minutes of perfusion, function continued to decline for hearts preperfused without CoA precursors, with the cardiac output falling to 53\% of the initial value and pressure-volume work declining to $46 \%$ of the initial value. If the precursors of $\mathrm{CoA}$ were included in the preperfusion media, both cardiac output and pressure-

Table II. Tissue Content of Key CoA and Carnitine Esters in Isolated Working Rat Hearts

\begin{tabular}{|c|c|c|c|c|c|}
\hline \multirow[b]{2}{*}{ Substrate } & \multirow{2}{*}{$\begin{array}{c}\text { Free } \\
\text { CoASH }\end{array}$} & \multicolumn{2}{|c|}{ CoA esters } & \multicolumn{2}{|c|}{ Carnitine esters } \\
\hline & & Acetyl & 3-Ketoacyl & Acetyl & 3-Ketoacyl \\
\hline & & & umol/g dry weight & & \\
\hline Acetoacetate (5 mM) & $0.136 \pm 0.035$ & $0.112 \pm 0.010$ & $0.060 \pm 0.012$ & $1.895 \pm 0.031$ & $0.030 \pm 0.010$ \\
\hline Acetoacetate + carnitine $(5 \mathrm{mM})$ & $0.213 \pm 0.044^{*}$ & $0.033 \pm 0.005^{*}$ & $0.122 \pm 0.027^{*}$ & $2.362 \pm 0.135^{*}$ & $0.273 \pm 0.084^{*}$ \\
\hline
\end{tabular}

Values are means \pm SEM for 10 hearts. $* P<0.05$ compared with acetoacetate alone. See text for further details. 
Table III. Tissue Content of COASH and Total CoA Immediately after Langendorf Perfusion Either without Substrate or with CoA Precursors

\begin{tabular}{llc}
\hline \multicolumn{1}{c}{ Substrates } & \multicolumn{1}{c}{ CoASH } & Total CoA \\
\hline & \multicolumn{2}{c}{ umol/g dry weight } \\
No precursors & $0.181 \pm 0.032$ & $0.567 \pm 0.031$ \\
CoA precursors & $0.353 \pm 0.013^{*}$ & $0.772 \pm 0.044^{*}$ \\
& & \\
\hline
\end{tabular}

Values are means \pm SEM for four hearts. ${ }^{*} P<0.05$. See text for further details.

volume work remained relatively stable for the duration of the perfusion, decreasing by only 8 and $14 \%$, respectively.

Preperfusion with CoA precursors resulted in an increase in the tissue content of CoASH after 30 minutes of perfusion with acetoacetate (Table IV), although the content was lower than the value immediately after the 45 -min preperfusion period. Likewise, CoASH content fell with the oxidation of acetoacetate in hearts that did not receive CoA supplementation. The total CoA pool remained elevated after 30 minutes of antero-

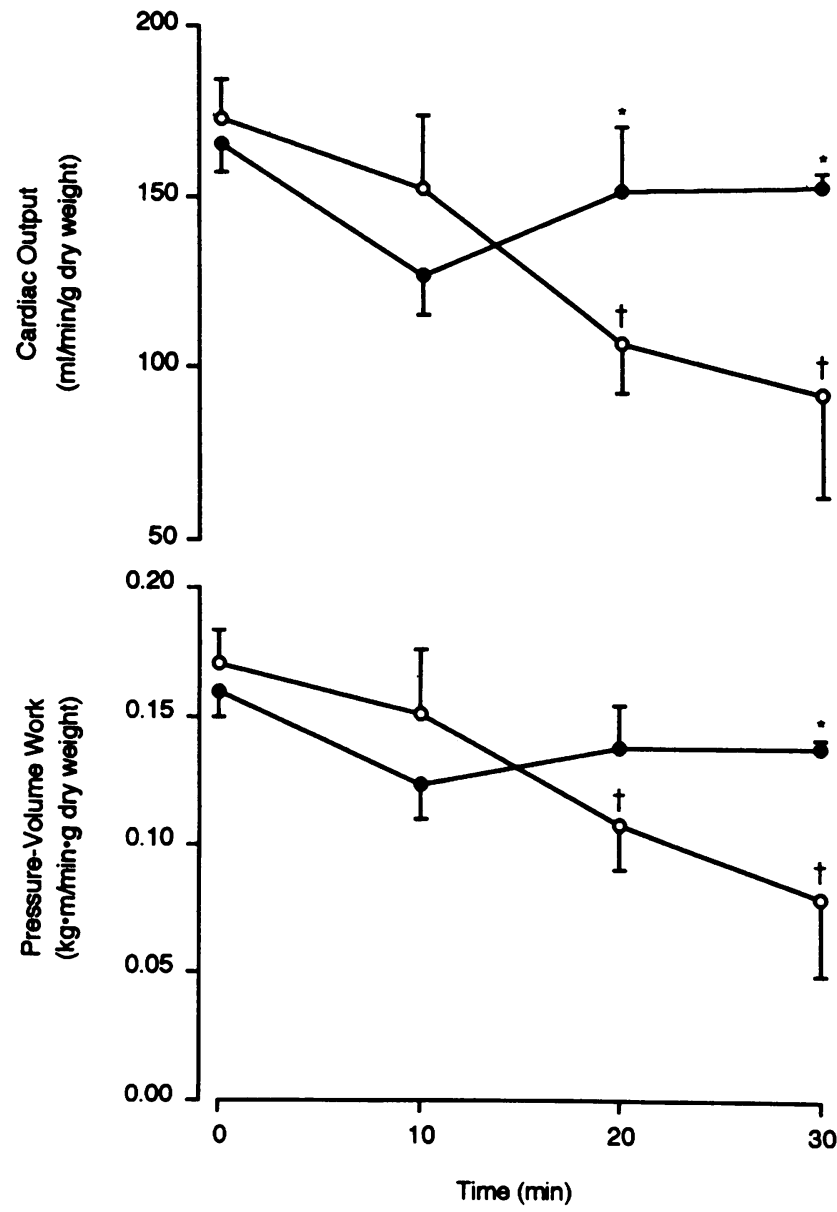

Figure 2. Cardiac output and pressure-volume work for working rat hearts $(n=5)$ oxidizing acetoacetate $(5 \mathrm{mM})$ after Langendorff preperfusion either with no substrates (o) or with $15 \mu \mathrm{M}$ pantothenic acid, $0.1 \mathrm{mM}$ cysteine, and $0.2 \mathrm{mM}$ DTT (๑). ${ }^{*} P<0.05$ compared with hearts preperfused with no substrates; ${ }^{\dagger} P<0.05$ compared with time zero for the respective group. grade perfusion in hearts that were preperfused with CoA precursors compared with those hearts that were preperfused without the CoA precursors. Similarly, the provision of CoA precursors during the preperfusion period resulted in a trend toward an increase in the acid-soluble 3-ketoacyl-CoA pool, the major component of which is assumed to be acetoacetylCoA under the substrate conditions used.

The provision of CoA precursors also lessened the inhibition of the 2-oxoglutarate dehydrogenase reaction. The relief of inhibition was suggested by a decrease in the tissue content of 2-oxoglutarate in hearts preperfused with the CoA precursors (Table IV) and an increase in the content of succinyl-CoA, the product of the 2-oxoglutarate dehydrogenase reaction, compared with hearts preperfused without substrates. Taken with the findings of increased CoASH and improved contractile function, these results indicate that availability of CoASH can indeed be a rate-limiting cofactor for the 2-oxoglutarate dehydrogenase reaction in heart muscle during the oxidation of ketone bodies.

\section{Discussion}

The above findings are consistent with the mechanism of sequestration of CoASH first described by Hülsmann et al. $(4,5)$ and further advanced by Taegtmeyer (1). In this mechanism, the activation of ketone bodies to acetoacetyl-CoA by 3-oxoacid-CoA transferase and subsequent cleavage to two molecules of acetyl-CoA by acetoacetyl-CoA thiolase depletes the intramitochondrial CoASH necessary to enter into the citric acid cycle reaction catalyzed by 2-oxoglutarate dehydrogenase. The fall in the tissue content of CoASH reported previously $(1$, 2 ) is mirrored in this study by a fall in the intramitochondrial [CoASH]. Further, the intramitochondrial [CoASH] during the oxidation of acetoacetate is close to the reported apparent $K_{\mathrm{mCoA}}$ for 2-oxoglutarate dehydrogenase (20). These studies are the first to demonstrate a fall in the intramitochondrial [CoASH] to a level consistent with inhibition of 2-oxoglutarate dehydrogenase. In further support of $\mathrm{CoASH}$ becoming a ratelimiting cofactor for 2-oxoglutarate dehydrogenase is our earlier observation that the fall in CoASH occurs after only 5 minutes of perfusion with acetoacetate as a sole substrate and is associated temporally with inhibition of 2-oxoglutarate dehydrogenase (2).

Limitation of CoASH has been implicated as a regulatory mechanism in several other pathways. In animal tissues, sequestration of CoASH has been suggested to result in inhibition of hepatic pyruvate carboxylase during the metabolism of sodium benzoate (23). Further, the inhibition of fatty acid oxidation by the action of 5-(tetradecyloxy)-2-furoic acid is thought to be mediated by decreasing the available CoASH (24). Sequestration of CoASH has even been implicated in the regulation of 2-oxoglutarate dehydrogenase and pyruvate dehydrogenase activities in plant mitochondria (25). Consistent with the findings of this study, it has been shown that partial protection against metabolic inhibition by erucic acid is afforded by elevated levels of CoA in brown adipose tissue (26, 27). In a situation somewhat reversed from those mentioned above and our current findings, it has been suggested that the hepatotoxic effects of valproic acid or other organic acids that require CoA activation can be reversed by the addition of parachlorobenzoic acid or para-nitrobenzoic acid, which compete with the toxic agents for $\operatorname{CoA}(28)$. 
Table IV. Tissue Metabolites after 30 min of Perfusion as Working Heart Preparations for Hearts Oxidizing Acetoacetate (5 mM) after Langendorff Preperfusion with No Substrates or with CoA Precursors

\begin{tabular}{lllcrrr}
\hline \multicolumn{1}{c}{ Substrates } & 2-Oxoglutarate & Succinyl-CoA & CoASH & Acetyl-CoA & 3-Ketoacyl-CoA & Total CoA \\
\hline & & \multicolumn{7}{c}{$\mu$ mollg dry weight } \\
No precursors & $0.340 \pm 0.053$ & $0.040 \pm 0.009$ & $0.125 \pm 0.017$ & $0.109 \pm 0.013$ & $0.054 \pm 0.004$ & $0.502 \pm 0.068$ \\
CoA precursors & $0.139 \pm 0.010^{*}$ & $0.069 \pm 0.005^{*}$ & $0.259 \pm 0.008^{*}$ & $0.103 \pm 0.012$ & $0.069 \pm 0.008$ & $0.762 \pm 0.072^{*}$
\end{tabular}

Values are means \pm SEM for five hearts. ${ }^{*} P<0.05$. See text for further details.

With the decrease in intramitochondrial [CoASH] confirmed, it was the second goal of these studies to determine whether it would be possible to enrich the mitochondrial pool of CoASH. Earlier investigators have shown that the addition of L-carnitine to mitochondrial suspensions results in the buffering of acyl-CoA derivatives (6). That is, there is an extramitochondrial accumulation of acetylcarnitine with a fall in the intramitochondrial concentration of acetyl-CoA and an increase in the intramitochondrial concentration of free CoASH. This study indicates that carnitine, at high extracellular concentrations, can indeed change the overall cellular distribution of short chain acyl groups between their $\mathrm{CoA}$ and carnitine derivatives, but this redistribution of acyl groups does not improve function during the oxidation of ketone bodies. This apparent lack of an effect by carnitine can be explained by the possibility that the exogenous carnitine does not have a signifcant effect on the intramitochondrial pool of CoASH. This notion is supported by the work of Brass and Hoppel, who demonstrated that the exchange between acylcarnitine and acyl-CoA pools is low in the liver (22) and that elevated levels of extramitochondrial carnitine do not affect intramitochondrial metabolism (21). It is therefore possible that there is insufficient equilibration of the exogenous carnitine with the mitochondrial pool of carnitine. While it is also possible that the addition of carnitine inhibited citric acid cycle flux by limiting the availability of acetyl-CoA to the citric acid cycle, this medianism is unlikely because the tissue content of acetyl-CoA in hearts perfused with acetoacetate and carnitine was similar to that in hearts that oxidized glucose and exhibited better function (data not presented).

In contrast to the findings with the addition of carnitine to hearts oxidizing acetoacetate, the supplementation of the available CoASH through the provision of CoA precursors resulted in an improvement in performance. Because it is the intramitochondrial pool of CoASH that is critical to 2-oxoglutarate dehydrogenase flux, the provision of CoA precursors must necessarily enrich this pool. In the present case, there is evidence to suggest that the provision of greater amounts of CoASH improves 2-oxoglutarate flux in the form of a decreased tissue content of 2-oxoglutarate and an increased tissue content of succinyl-CoA. While the initial steps of CoA synthesis occur in the cytosol, the conversion of 4'-phosphopantetheine to dephosphocoenzyme A and finally to CoA can occur in both the cytosol and mitochondria (29-31). Therefore, the addition of exogenous precursors of $\mathrm{CoA}$ can affect the intramitochondrial pool of CoA.

It is interesting to note that the CoA content of the rat heart is decreased by insulin (32) but increased by either acute streptozotocin diabetes or fasting (33). These changes in CoA are to be expected because of the greater dependence on fatty acids and ketone bodies in diabetes and fasting. However, it has also been shown that the phosphorylation of pantothenic acid to 4'-phosphopantothenic acid, the rate-limiting step of CoA synthesis, is depressed in diabetic hearts (34) and inhibited by the ketone body $\beta$-hydroxybutyrate (35). Further investigation is necessary to determine the effects of diabetes on ketone bodymediated failure in the heart. Specifically, is the diabetic heart more resistant to ketone body-mediated failure by virtue of an increased content of $\mathrm{CoA}$, or does the inhibition of pantothenate kinase (both by the diabetic state and by the provision of ketone bodies as a significant energy substrate) accelerate the inhibition of citric acid cycle flux and contractile failure?

In summary, the metabolism of ketone bodies in rat heart results in inhibition of citric acid cycle flux at the level of 2oxoglutarate dehydrogenase through limitation of the intramitochondrial supply of the required cofactor CoASH. This limitation results from the sequestration of $\mathrm{CoASH}$ as the thioesters acetoacetyl-CoA and acetyl-CoA. While the addition of L-carnitine does not improve function for hearts oxidizing acetoacetate, it is possible that there is not an adequate equilibration of the exogenous pool of carnitine with the intramitochondrial carnitine, such that not enough CoASH was made available to enter into the 2-oxoglutarate dehydrogenase reaction. However, enrichment of the supply of CoA and CoASH in the heart both reverses the inhibition of 2-oxoglutarate dehydrogenase and improves contractile performance during the oxidation of ketone bodies. These findings underscore the fact that the supply of CoASH is central to ketone body metabolism and proper citric acid cycle functioning. Because ketone bodies represent a significant energy source for the heart, especially during fasting and diabetic ketoacidosis, it is important to understand the regulation of ketone body metabolism as well as the effects of ketone body oxidation on citric acid cycle functioning and, ultimately, the functioning of the heart.

\section{Acknowledgments}

This study was supported in part by a grant-in-aid from the American Heart Association (National Center). Dr. Russell was supported by the $\mathrm{MD} / \mathrm{PhD}$ program cosponsored by The University of Texas Medical School at Houston and The University of Texas M. D. Anderson Cancer Center.

\section{References}

1. Taegtmeyer, H. 1983. On the inability of ketone bodies to serve as the only energy providing substrate for rat heart at physiological work load. Basic Res. Cardiol. 78:435-450.

2. Russell, R. R., and H. Taegtmeyer. 1991. Changes in citric acid cycle flux and anaplerosis antedate the functional decline in isolated rat hearts utilizing acetoacetate. J. Clin. Invest. 87:384-390.

3. Williamson, J. R., and H. A. Krebs. 1961. Acetoacetate as fuel of respiration in the perfused rat heart. Biochem. J. 80:540-547. 
4. Hülsmann, W. C., D. Siliprandi, M. Ciman, and N. Siliprandi. 1964. Effect of carnitine on the oxidation of a-oxoglutarate to succinate in the presence of acetoacetate or pyruvate. Biochim. Biophys. Acta. 93:166-168.

5. Hülsmann, W. C., E. M. Wit-Peeters, and C. Benkhuysen. 1966. Factors influencing fatty acid metabolism in mitochondria. In Regulation of Metabolic Processes in Mitochondria. J. M. Tager, S. Papa, E. Quagliariello, and E. C. Slater, editors. Elsevier Publishing Co., New York. 460-475.

6. Idell-Wenger, J. A., L. W. Grotyohann, and J. R. Neely. 1982. Regulation of fatty acid utilization in heart: role of the carnitine-acetyl-CoA transferase and carnitine-acetyl carnitine translocase system. J. Mol. Cell. Cardiol. 14:413-417.

7. Vary, T. C., and J. R. Neely. 1983. Sodium dependence of carnitine transport in isolated perfused adult rat hearts. Am. J. Physiol. 244:H247-H252.

8. Robishaw, J. D., and J. R. Neely. 1984. Pantothenate kinase and control of CoA synthesis in heart. Am. J. Physiol. 246:H532-H541.

9. Lopaschuk, G. D., C. A. Hansen, and J. R. Neely. 1986. Fatty acid metabolism in hearts containing elevated levels of CoA. Am. J. Physiol. 250:H351H359.

10. Taegtmeyer, H., R. Hems, and H. A. Krebs. 1980. Utilization of energy providing substrates in the isolated working rat heart. Biochem. J. 186:701-711.

11. Langendorff, O. 1895. Untersuchungen am überlebenden Säugethierherzen. Arch. Gesamte Physiol. Mens. Tiere (Pfluegers). 61:291-332.

12. Chua, B. H. L., K. E. Giger, B. J. Kleinhans, J. D. Robishaw, and H. E Morgan. 1984. Differential effects of cysteine on protein and coenzyme A synthesis in rat heart. Am. J. Physiol. 247:C99-C106.

13. Idell-Wenger, J. A., L. W. Grotyohann, and J. R. Neely. 1982. An improved method for isolation of mitochondria in high yields from normal, ischemic and autolyzed rat hearts. Anal. Biochem. 125:269-276.

14. LaNoue, K. F., E. I. Walajtys, and J. R. Williamson. 1973. Regulation of glutamate metabolism and interaction with the citric acid cycle in rat heart mitochondria. J. Biol. Chem. 248:7171-7183.

15. Williamson, J. R., and B. E. Corkey. 1967. Assays of intermediates of the citric acid cycle and related compounds by fluorometric enzyme methods. Methods Enzymol. 13:434-512.

16. Kauppinen, R. A., J. K. Hiltunen, and I. E. Hassinen. 1980. Subcellular distribution of phosphagens in isolated perfused rat heart. FEBS (Fed. Eur. Bio chem. Soc.) Lett. 112:273-276.

17. Chance, B., and G. R. Williams. 1955. Respiratory enzymes in oxidative phosphorylation. III. The steady state. J. Biol. Chem. 217:409-427.

18. Gornal, A. G., C. J. Bardawill, and M. M. David. 1949. Determination of serum proteins by means of the Biuret reaction. J. Biol. Chem. 177:751-766.

19. Unitt, J. F., J. G. McCormack, D., Reid, L. K. MacLachlan, and P. J. England. 1989. Direct evidence for a role of intramitochondrial $\mathrm{Ca}^{2+}$ in the regulation of oxidative phosphorylation in the stimulated rat heart. Biochem. J. 262:1293-1301.
20. Read, G., B. Crabtree, and G. H. Smith. 1977. The activities of 2-oxoglutarate dehydrogenase and pyruvate dehydrogenase in hearts and mammary glands from ruminants and non-ruminants. Biochem. J. 164:349-355.

21. Brass, E. P., and C. L. Hoppel. 1980. Effect of carnitine on mitochondrial oxidation of palmitoylcarnitine. Biochem. J. 188:451-458.

22. Brass, E. P., and C. L. Hoppel. 1980. Relationship between acid-soluble carnitine and coenzyme A pools in vivo. Biochem. J. 190:495-504.

23. Griffith, A. D., D. M. Cyr, S. G. Egan, and G. C. Tremblay. 1989. Inhibition of pyruvate carboxylase by sequestration of coenzyme A with sodium benzoate. Arch. Biochem. Biophys. 269:201-207.

24. Otto, D. A., C. Chatzidakis, E. Kasziba, and G. A. Cook. 1985. Reciprocal effects of 5-(tetradecyloxy)-2-furoic acid on fatty acid oxidation. Arch. Biochem. Biophys. 242:23-31.

25. Dry, I. B., and J. T. Wiskich. 1987. 2-Oxoglutarate dehydrogenase and pyruvate dehydrogenase activities in plant mitochondria: interaction via a common coenzyme A pool. Arch. Biochem. Biophys. 257:92-99.

26. Alexson, S., and B. Cannon. 1985. Inhibition of acetyl-carnitine oxidation by erucoyl-carnitine in rat brown-adipose-tissue mitochondria is due to $\mathrm{CoA}$ sequestration. Biochim. Biophys. Acta. 834:149-158.

27. Alexson, S., J. Nedergaard, and B. Cannon. 1986. Partial protection against erucoyl-carnitine inhibition in hamster brown-adipose-tissue mitochondria is due to high CoA levels: a comparison with rat brown-adipose-tissue mitochondria. Comp. Biochem. Physiol. B Comp. Biochem. 83:191-196.

28. Swartzentruber, M. S., and R. A. Harris. 1987. Inhibition of metabolic processes by coenzyme-A-sequestering aromatic acids. Prevention by parachloro- and para-nitrobenzoic acids. Biochem. Pharmacol. 36:3147-3153.

29. Hoaglund, M., and G. Novelli. 1954. Biosynthesis of coenzyme A from phosphopantetheine and of pantetheine from pantothenate. J. Biol. Chem. 207:767-773

30. Akibo, Y. 1967. Investigations on pantothenic acid and its related compounds. X. Biochemical studies. 5. Purification and substrate specificity of pantothenoylcysteine decarboxylase from rat liver. J. Biochem. 61:300-308.

31. Tahiliani, A. G., and J. R. Neely. 1987. Mitochondrial synthesis of coenzyme A is on the external surface. J. Mol. Cell. Cardiol. 19:1161-1167.

32. Neely, J. R., J. D. Robishaw, and T. C. Vary. 1982. Control of myocardial levels of CoA and carnitine. J. Mol. Cell. Cardiol. 14(Suppl. 3):37-42

33. Reibel, D. K., B. W. Wyse, D. A. Berkich, W. M. Palko, and J. R. Neely. 1981. Effects of diabetes and fasting on pantothenic acid metabolism in rats. Am. J. Physiol. 240:E597-E601.

34. Beinlich, C. J., J. D. Robishaw, and J. R. Neely. 1989. Metabolism of pantothenic acid in hearts of diabetic rats. J. Mol. Cell. Cardiol. 21:641-649.

35. Robishaw, J. D., D. Berkich, and J. R. Neely. 1982. Rate-limiting step and control of coenzyme A synthesis in cardiac muscle. J. Biol. Chem. 257:1096710972. 